\title{
Tomatidine and analog FC04-100 possess bactericidal activities against Listeria, Bacillus and Staphylococcus spp
}

\author{
Isabelle Guay ${ }^{1 \dagger}$, Simon Boulanger ${ }^{1 \dagger}$, Charles Isabelle ${ }^{1}$, Eric Brouillette ${ }^{1}$, Félix Chagnon ${ }^{2}$, Kamal Bouarab ${ }^{1}$,
} Eric Marsault ${ }^{2^{*}}$ and François Malouin ${ }^{1 *}$ (i)

\begin{abstract}
Background: Tomatidine (TO) is a plant steroidal alkaloid that possesses an antibacterial activity against the small colony variants (SCVs) of Staphylococcus aureus. We report here the spectrum of activity of TO against other species of the Bacillales and the improved antibacterial activity of a chemically-modified TO derivative (FC04-100) against Listeria monocytogenes and antibiotic multi-resistant S. aureus (MRSA), two notoriously difficult-to-kill microorganisms.
\end{abstract}

Methods: Bacillus and Listeria SCVs were isolated using a gentamicin selection pressure. Minimal inhibitory concentrations (MICS) of TO and FC04-100 were determined by a broth microdilution technique. The bactericidal activity of TO and FC04-100 used alone or in combination with an aminoglycoside against planktonic bacteria was determined in broth or against bacteria embedded in pre-formed biofilms by using the Calgary Biofilm Device. Killing of intracellular SCVs was determined in a model with polarized pulmonary cells.

Results: TO showed a bactericidal activity against SCVs of Staphylococcus aureus, Bacillus cereus, B. subtilis and Listeria monocytogenes with MICs of $0.03-0.12 \mu \mathrm{g} / \mathrm{mL}$. The combination of an aminoglycoside and TO generated an antibacterial synergy against their normal phenotype. In contrast to TO, which has no relevant activity by itself against Bacillales of the normal phenotype (MIC > $64 \mu \mathrm{g} / \mathrm{mL}$ ), the TO analog FC04-100 showed a MIC of 8-32 $\mu \mathrm{g} / \mathrm{mL}$. Furthermore, FC04-100 showed a strong bactericidal activity against L. monocytogenes SCVs in kill kinetics experiments, while TO did not. The addition of FC04-100 (4 $\mu \mathrm{g} / \mathrm{mL})$ to a cefalexin:kanamycin (3:2) combination improved the activity of the combination by 32 fold against cefalexin and kanamycin-resistant MRSA strains. In combination with gentamicin, FC04-100 also exhibited a strong bactericidal activity against biofilm-embedded S. aureus. Also, FC04-100 and TO showed comparable intracellular killing of S. aureus SCVs.

Conclusions: Chemical modifications of TO allowed improvement of its antibacterial activity against prototypical $S$. aureus and of its bactericidal activity against $L$. monocytogenes. Antibacterial activities against such prominent pathogens could be useful to prevent Listeria contamination in the food chain or as treatment for MRSA infections.

Keywords: Tomatidine, Aminoglycoside, Synergy, SCV, Bacillales, S. aureus, L. monocytogenes, Foodborne disease, Cystic fibrosis

\footnotetext{
* Correspondence: Eric.Marsault@USherbrooke.ca;

Francois.Malouin@USherbrooke.ca

${ }^{\dagger}$ Equal contributors

Département de pharmacologie, Faculté de médecine et des sciences de la

santé, Université de Sherbrooke, 3001, 12 th avenue Nord, Sherbrooke, QC

J1H 5N4, Canada

${ }^{1}$ Centre d'Étude et de Valorisation de la Diversité Microbienne (CEVDM),

Département de biologie, Faculté des sciences, Université de Sherbrooke,

2500 Boul. Université, Sherbrooke, QC J1K 2R1, Canada
}

C The Author(s). 2018 Open Access This article is distributed under the terms of the Creative Commons Attribution 4.0 International License (http://creativecommons.org/licenses/by/4.0/), which permits unrestricted use, distribution, and reproduction in any medium, provided you give appropriate credit to the original author(s) and the source, provide a link to the Creative Commons license, and indicate if changes were made. The Creative Commons Public Domain Dedication waiver (http://creativecommons.org/publicdomain/zero/1.0/) applies to the data made available in this article, unless otherwise stated. 


\section{Background}

The Bacillales are divided into the genus of Staphylococcus, Listeria and Bacillus. A number of bacterial species such as Listeria spp. and Bacillus spp. can contaminate food and cause infections in humans [1]. To name a few, Listeria monocytogenes, L. ivanovii, and Bacillus cereus can cause listeriosis [2] and food poisoning [3]. Bacillus subtilis, B. coagulans, B. licheniformis and B. sphaericus are also known to cause illnesses. Bacillus anthracis causes anthrax and can often be acquired by contact with food producing animals and cattle (beef cattle, sheep, etc.) and this bacterium is also well-known for its endospores that have been used as biological weapons [4]. Staphylococci are divided in coagulase-positive species, Staphylococcus aureus being the most clinically relevant of this group, and coagulasenegative species, such as $S$. epidermidis, the most prevalent pathogen associated with infections of implanted medical devices [5]. The emergence and spread of resistance to multiple antibiotics in staphylococci is now considered a real health treat and impaired therapeutic endeavor to combat these bacteria [6]. Notably, the prevalence of methicillin-resistant $S$. aureus (MRSA) has steadily increased over the recent years, not only in hospitals but also in the community [7], and in veterinary medicine and livestock [8-10].

Staphylococcus aureus small-colony variants (SCVs) have attracted a great deal of interest over the past recent years. S. aureus SCVs often present a dysfunctional oxidative metabolism causing a slow growth and a change in the expression of virulence factors [11]. This dysfunctional oxidative metabolism is also responsible for a decreased susceptibility to aminoglycoside antibiotics because this class of molecules requires the proton-motive force in order to penetrate the bacterium [12]. This respiratory deficiency is often caused by mutations affecting the electron transport system, and several SCV isolates can recover normal growth with supplemental hemin or menadione, which are needed to synthesize electron transport system components. S. aureus SCVs often are isolated from chronic infections, such as lung infections in cystic fibrosis (CF) patients, osteomyelitis, septic arthritis, bovine mastitis, and from infections associated with orthopedic devices [11, 13, 14]. The ability of $S$. aureus to switch back and forth from the prototypic to the SCV phenotypes in vivo is an integral part of the pathogenesis of $S$. aureus and may be responsible for the establishment of chronic infections $[15,16]$.

Tomatidine (TO) is a steroidal alkaloid produced by the Solanaceae plant family such as the tomato $[17,18]$. We showed previously the antibacterial activity of TO against $S$. aureus SCVs and also documented a strong synergic activity of TO in combination with aminoglycoside antibiotics against prototypic $S$. aureus [19, 20]. Recently, we synthesized a variety of TO analogs in order to explore the structure-activity relationship of this new class of antibiotics able to act on SCVs [21]. One analog, FC04-100, showed the same steroidal backbone as the natural molecule but with an additional carbon chain and amines on the A cycle (Fig. 1). Preliminary characterization of FC04-100 revealed an antibacterial activity that was similar to that of TO against $S$. aureus SCVs and also preserved the synergic activity with aminoglycosides against prototypic S. aureus [21]. However, contrary to TO, FC04-100 showed notable antibiotic activity by itself against prototypic S. aureus with a MIC of $8-16 \mu \mathrm{g} / \mathrm{mL}$, whereas that of TO was $>64 \mu \mathrm{g} / \mathrm{mL}$.

The aim of this study was to further describe the spectrum of activity of TO and FC04-100. It was of great interest to see if the discovered antibacterial activity of FC04-100 against prototypical S. aureus modifies or improves its spectrum of antibacterial activity. The susceptibility of several species among the Bacillales to TO and FC04-100 alone or in combination with other antibiotics was investigated. The improved antibacterial activity of FC04-100 was demonstrated against Listeria monocytogenes and methicillin-resistant $S$. aureus (MRSA).

\section{Methods}

\section{Chemical reagents and antibiotics}

Tomatidine (TO) (Sigma Aldrich, Oakville, Ontario, Canada) (Fig. 1a) was solubilised in dimethylsulfoxide (DMSO) at a concentration of $2 \mathrm{mg} / \mathrm{mL}$. The TO analog, FC04-100 (Fig. 1b) was synthesized [21] and solubilised in DMSO at $20 \mathrm{mg} / \mathrm{mL}$. Cefalexin, kanamycin, gentamicin (GEN) (all from Sigma Aldrich), were solubilised in water at a concentration of $10 \mathrm{mg} / \mathrm{mL}$. Menadione was solubilized in DMSO, hemin in 1.4 $\mathrm{M} \mathrm{NH}_{4} \mathrm{OH}$ and thymidine in water (all from Sigma Aldrich) and were prepared at a concentration of $10 \mathrm{mg} / \mathrm{mL}$.

\section{Bacterial strains and growth conditions}

Staphylococcus aureus ATCC 29213, S. aureus Newbould (ATCC 29740), S. epidermidis ATCC 12228, Listeria monocytogenes ATCC 13932, Bacillus subtilis ATCC 6333 and Bacillus cereus ATCC 11778 were used as prototypic strains. Methicillin-resistant $S$. aureus (MRSA) strains COL and USA100 (hospital acquiredMRSA, ATCC BAA-41) and strain USA300 (community acquired-MRSA, ATCC BAA-1556), were also used in this study. The reference laboratory strain S. aureus SH1000, an isogenic mutant strain derived from $S$. aureus 8523-4 but with a functional rsbU allele [22], was used for its good yield of biofilm production. The $S$. aureus strains CF07-L (prototypic) and CF07-S (SCV phenotype) are genetically related clinical strains originally isolated from a cystic fibrosis patient [19]. Strain CF07-S was used in the intracellular infection model. Staphylococcus and Bacillus 


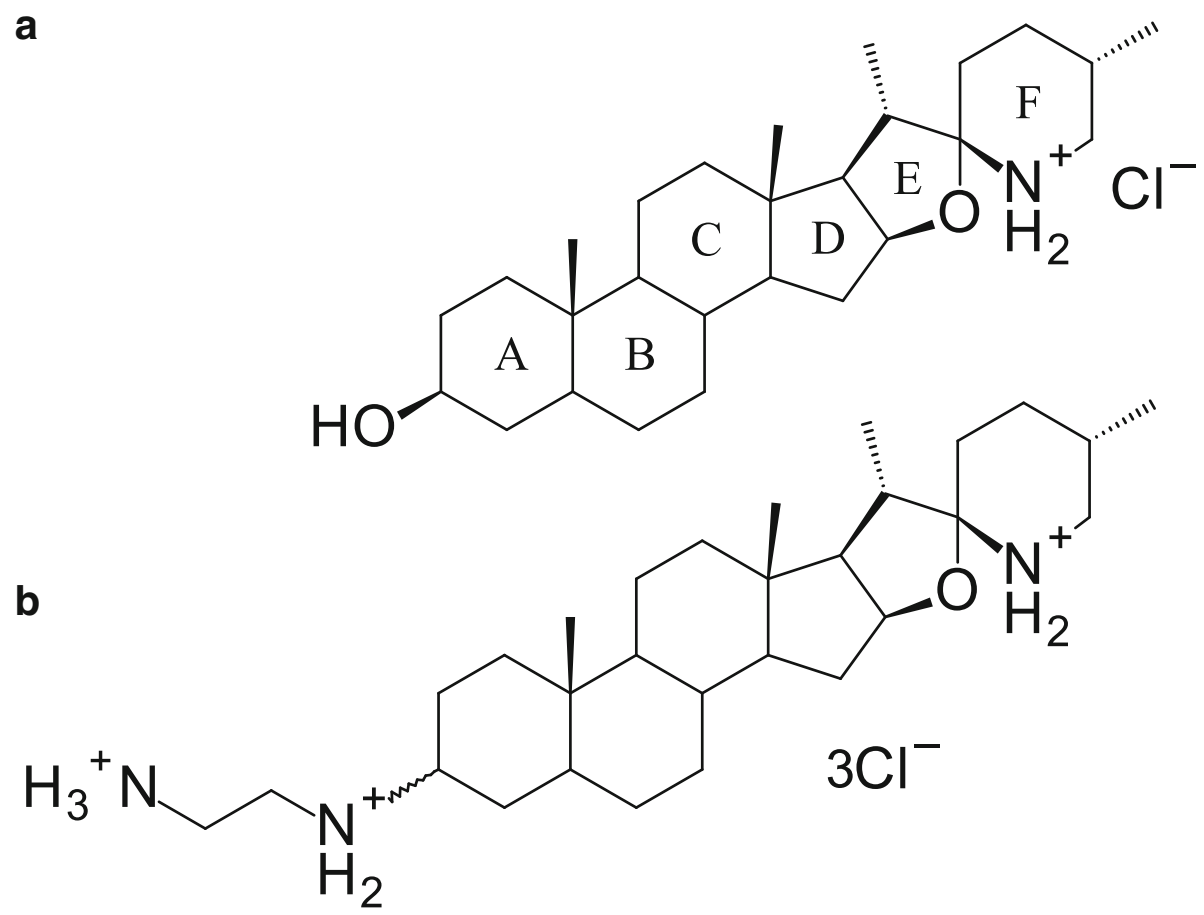

Fig. 1 Tomatidine (a) is a steroid alkaloid structurally characterized by 6 rings, 12 stereogenic centers, a 3 -hydroxyl group and spiro-fused E, F rings in the form of an aminoketal. The tomatidine analog, FC04-100 (b) contains a diamine in position 3 of ring A

were maintained on Tryptic Soy agar (TSA, BD, Mississauga, Ontario, Canada), whereas L. monocytogenes was grown on brain-heart infusion (BHI) agar (BD, Mississauga, Ontario, Canada).

\section{Small colony variants (SCVs)}

For $S$. aureus, strain Newbould $\Delta h e m B$ was used as the reference SCV. Newbould $\triangle$ hemB was generated from strain Newbould (ATCC 29740) by disrupting the hemB gene with the $\operatorname{erm} A$ cassette by homologous recombination [23]. Another SCV hemB mutant was similarly constructed from S. aureus ATCC 29213 [24]. SCVs from B. cereus, B. subtillis and L. monocytogenes were generated by growth in presence of a subinhibitory concentration of GEN. Briefly, overnight broth cultures (18-20 h) were used to inoculate BHI broths at a dilution of $1: 100$, supplemented or not with 0.25 to $1 \mathrm{X}$ the MIC of GEN. Cultures were incubated $18 \mathrm{~h}$ at $35{ }^{\circ} \mathrm{C}$ with shaking (225 rpm) and then adjusted to an $A_{595} \mathrm{~nm}$ of 2.0 in PBS at $4{ }^{\circ} \mathrm{C}$. Determination of CFU and SCV colonies was done by serial dilution plating. SCVs were obtained by plating on TSA (BHI agar for L. monocytogenes) containing GEN at a concentration of 8 to 16 times the induction concentration followed by an incubation of $48 \mathrm{~h}$ at $35{ }^{\circ} \mathrm{C}$. The pinpoint colonies selected by this method were confirmed to be SCVs by streaking several of them on agar plates without antibiotic. SCV that conserved their phenotype after two passages were considered to have a stable phenotype and were used in subsequent experiments. Selected SCVs had a colony size a tenth of the size of a normal colony and showed no pigmentation or hemolytic activity on blood agar plates. The SCV species were confirmed by rDNA $16 \mathrm{~S}$ sequencing after PCR amplification using universal primers [25].

\section{Auxotrophy assays}

For SCVs, auxotrophism is defined as the requirement of specific compounds in order to regain a normal growth phenotype [26]. An agar diffusion method was used to characterize the auxotrophism of SCVs using hemin or menadione $(1-10 \mu \mathrm{g}$ each/well) or thymidine (100 $\mathrm{\mu g} /$ well) on an inoculated Mueller-Hinton agar (MHA) or Brain-heart infusion agar (BHIA) plate [27]. Auxotrophy for specific supplements was detected by a zone of normal growth surrounding the well after $18 \mathrm{~h}$ of incubation at $35{ }^{\circ} \mathrm{C}$. The results were confirmed by two independent experiments.

\section{Antibiotic susceptibility testing}

MICs were determined by a broth microdilution technique following the recommendations of the Clinical and Laboratory Standards Institute (CLSI) [28], except that the incubation period was extended to $48 \mathrm{~h}$ and the medium used was BHI to allow SCVs to reach maximal growth as previously described [29]. As recommended 
by the CLSI, $L$. monocytogenes (and its SCVs) were grown in cation-adjusted Muller-Hinton broth (CAMHB, BD) containing 3\% lysed horse blood (LHB, Remel, Hartford, CT, USA). The reported results were obtained from three independent experiments.

\section{Time-kill experiments}

Kill kinetics were performed to determine whether the effect of compounds alone or in combination with an aminoglycoside was bacteriostatic or bactericidal. Bacteria were inoculated at $10^{5}-10^{6} \mathrm{CFU} / \mathrm{mL}$ in the appropriate medium in the absence or presence of the different antibiotic compounds at concentrations specified in figure legends. At several points in time at $35{ }^{\circ} \mathrm{C}$ (225 rpm), bacteria were sampled, serially diluted, and plated on TSA for CFU determinations. Plates were incubated for 24 or $48 \mathrm{~h}$ at $35{ }^{\circ} \mathrm{C}$ for normal and SCV strains, respectively. The data were collected from a minimum of three independents experiments.

\section{Antibiotic activity in biofilms}

The viability of bacteria in biofilms was evaluated based on previously described protocols [30-32]. For these assays, 96-peg lids and corresponding 96-well plates were used (Thermo Scientific, Ottawa, ON, Canada). Wells were inoculated with a suspension of $S$. aureus SH-1000 adjusted to a 0.5 McFarland standard in TSB $(150 \mu \mathrm{L}$ per wells) and plates with 96-well lids were incubated at $35{ }^{\circ} \mathrm{C}$ with an agitation of $120 \mathrm{rpm}$ for $24 \mathrm{~h}$. The biofilms on pegs were then washed three times with $200 \mu \mathrm{L}$ PBS. Biofilms on pegs were further incubated in fresh 96-well plates containing $200 \mu \mathrm{L}$ of TSB containing or not antibiotic compounds or a combination of molecules at $35{ }^{\circ} \mathrm{C}, 120 \mathrm{rpm}$ for $24 \mathrm{~h}$. The treated biofilms on pegs were washed three times in PBS and bacteria in biofilms were recovered by sonication in a new microtiter plate containing $200 \mu \mathrm{L}$ of PBS per wells using an ultrasonicator bath for $10 \mathrm{~min}$ followed by centrifugation for $5 \mathrm{~min}$ at 1000 RPM. The bacteria recovered in each well were suspended, serially diluted, plated on TSA and incubated at $35{ }^{\circ} \mathrm{C}$ for $24 \mathrm{~h}$ before $\mathrm{CFU}$ determination.

\section{Cell invasion and measurement of intracellular antibiotic activity}

The Calu-3 cell line (ATCC HTB 55, Homo sapiens lung adenocarcinoma), was cultured in Eagle's Minimum Essential Medium (EMEM) supplemented with $0.1 \mathrm{mM}$ MEM nonessential amino acids, $1 \mathrm{mM}$ of sodium pyruvate, $100 \mathrm{U} / \mathrm{mL}$ penicillin, $100 \mu \mathrm{g} / \mathrm{mL}$ streptomycin, $2.5 \mu \mathrm{g} / \mathrm{mL}$ of Fungizone and $10 \%$ fetal bovine serum (FBS) at $37{ }^{\circ} \mathrm{C}$ in $5 \% \mathrm{CO}_{2}$. For routine culture, $4 \mu \mathrm{g} / \mathrm{mL}$ of puromycin was added to culture media. All cell culture reagents were purchased from Wisent (St-Bruno, QC, Canada).
The cell invasion assay was performed with the Calu-3 cells in an air-liquid interface as previously described with few modifications [19]. Briefly, cells were seeded at $1.5 \times 10^{5}$ cells per insert in a 12-well Transwell plate (Corning, Tewksbury, MA) and cultured for 10 days with the apical medium replaced each day. The complete medium in the basal compartment was replaced by the invasion medium (1\% FBS and no antibiotics) $18 \mathrm{~h}$ before invasion assays. Inocula were prepared by suspending bacteria grown $20 \mathrm{~h}$ on BHI agar plates in ice-cold PBS. Bacteria were then washed three times in ice-cold PBS and suspended in the invasion medium supplemented with $0.5 \%$ BSA at a density of approximately $4.0 \times 10^{8} \mathrm{CFU} / \mathrm{mL}$. Cells were washed twice with PBS and $250 \mu \mathrm{L}$ of bacterial suspension (multiplicity of infection [MOI] of 10, i.e., the ratio bacteria/cells) were apically added to each insert. Invasion of cells by bacteria was allowed for $3 \mathrm{~h}$, inserts were emptied and washed three times with PBS. The basal medium was changed for the invasion medium with $20 \mu \mathrm{g} / \mathrm{mL}$ of lysostaphin (Sigma) to kill extracellular bacteria and with or without the tested antibiotic compounds. The infected cells were incubated for a total of $48 \mathrm{~h}$ with a change of medium at $23 \mathrm{~h}$ (invasion medium with lysostaphin with or without the tested antibiotic compounds). The invasion medium with lysostaphin but without tested compound was also apically added $1 \mathrm{~h}$ before cell lysis to ensure that only intracellular bacteria were counted. At $48 \mathrm{~h}$, following three washes with PBS, the apical and basal media were removed and cells were detached with $100 \mu \mathrm{L}$ of trypsin $0.25 \%$ and lysed for $10 \mathrm{~min}$ by the addition of $400 \mu \mathrm{L}$ of water containing $0.05 \%$ of Triton X-100. Then, $50 \mu \mathrm{L}$ of PBS (10X) was added and mixed. Lysates were serially diluted 10-fold and plated on TSA for CFU determination. Plates were incubated at $35^{\circ} \mathrm{C}$ for $48 \mathrm{~h}$.

\section{Statistical analysis}

Statistical analyses were carried out with the GraphPad Prism software (v.6.02). Bacterial CFUs were transformed in base 10 logarithm values before being used for statistical analyses. One-way ANOVA and post-tests were used as appropriate for the analysis of data as specified in each of the figure legends.

\section{Results}

\section{Emergence the SCV phenotype}

The SCV phenotype is characterized by a slow growth yielding a small colony size on agar plates (i.e., typically $1 / 5$ to $1 / 10$ of the normal colony size). Two prototypic S. aureus, Newbould and CF07-L, and their SCV counterparts, Newbould $\Delta$ hemB and CF07-S, respectively, were used as control strains (Fig. 2a and b). Figure 2 shows the colony sizes of the parental strains compared to that of the SCV derivatives. A subinhibitory 

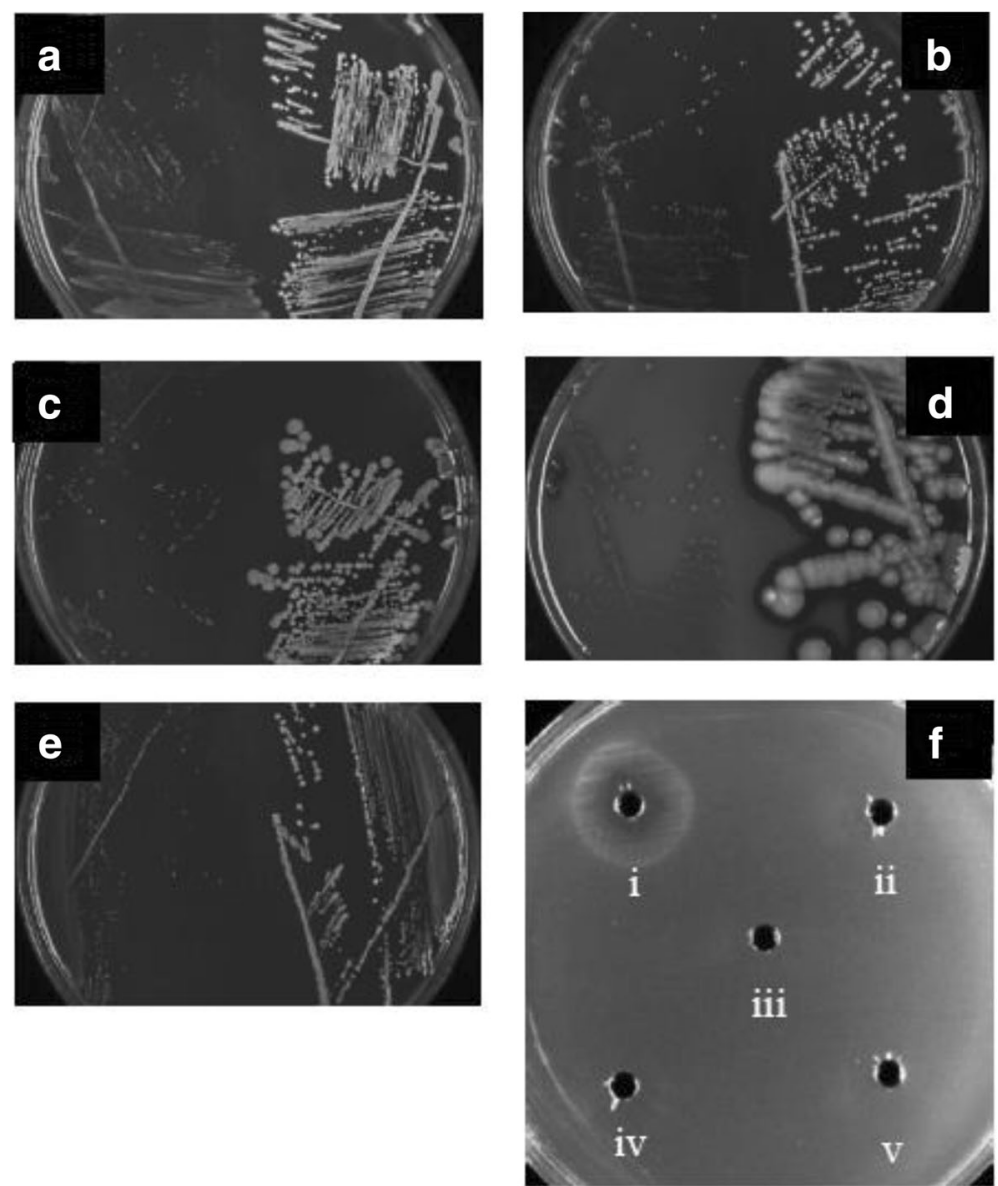

Fig. 2 Typical colony sizes for SCV and prototypic strains (left and right sides of the plates, respectively) for (a) S. aureus CF07-S and CF07-L, (b) S. aureus Newbould $\Delta$ hemB and Newbould, (c) B. cereus ATCC 11778 (SCV\#1 and prototypic), (d) B. subtilis ATCC 6333 (SCV\#2 and prototypic), and (e) L. monocytogenes ATCC 13932 (SCV\#1 and prototypic). In (f), a TSA plate was inoculated with L. monocytogenes ATCC 13932 SCV\#2 and wells were filled with (i) $10 \mu \mathrm{g}$ hemin, (ii) $10 \mu \mathrm{g}$ menadione, (iii) $100 \mu \mathrm{g}$ thymidine, and diluents (iv) DMSO, and (v) $\mathrm{NH}_{4} \mathrm{OH} 1.4 \mathrm{~N}$. A zone of enhanced growth is observed around (i). The enhanced growth appears white and surrounds a small zone of growth inhibition

concentration of GEN was used to promote the emergence of the SCV phenotype for B. cereus, B. subtilis and $L$. monocytogenes. The $B$. cereus SCVs were obtained at an inducing concentration of $1 \mathrm{X}$ MIC of GEN (Fig. 2c), B. subtilis SCVs at $0.5 \mathrm{X}$ MIC (Fig. 2d), and L. monocytogenes SCVs at $0.25 \mathrm{X}$ MIC (Fig. 2e). The SCV isolates selected for the rest of the study were stable and kept their small-colony phenotype without a GEN selection pressure. The B. cereus SCVs showed auxotrophy for menadione (Table 1 ), some of the L. monocytogenes SCVs showed auxotrophy for hemin (Fig. 2f, Table 1), while the B. subtilis SCVs showed no apparent auxotrophy to hemin, menadione or thymidine (i.e., unknown auxotrophy, Table 1). These results show that SCVs with defects in their respiratory chain (i.e., hemin or menadione auxotrophy) can indeed emerge from a selective pressure with aminoglycosides.

\section{Antibacterial activity of TO and analog FCO4-100 against Bacillales spp}

The MICs of TO and FC04-100 against a panel of prototypic and SCV strains of the Bacillales are reported in Table 1. The MICs for TO ranged from 0.03 to $0.12 \mu \mathrm{g} / \mathrm{mL}$ against all the SCVs tested from the species $S$. aureus, L. monocytogenes, B. cereus and B. subtilis, whereas TO showed no activity (MIC $>64 \mu \mathrm{g} / \mathrm{mL}$ ) against the prototypic strains including $S$. epidermidis (Table 1). Noteworthy, strains of the normal phenotype were more susceptible to GEN compared to their SCV derivatives; this was expected because aminoglycosides (e.g., GEN) require an active respiratory chain and the proton-motive force in order to penetrate the bacterium. Besides, the MICs of analog FC04-100 against the SCV strains (MIC of $1-2 \mu \mathrm{g} / \mathrm{mL}$, Table 1 ) were slightly higher than that found for TO. On the other hand, the MICs of 
Table 1 Susceptibility of prototypic and SCV strains and species to TO, FC04-100, GEN or the combination of GEN with one of either steroidal alkaloids

\begin{tabular}{|c|c|c|c|c|c|c|}
\hline \multirow[b]{2}{*}{ Species and strains } & \multirow[b]{2}{*}{$\operatorname{Auxo}^{a}$} & \multicolumn{5}{|c|}{$\mathrm{MIC}(\mu \mathrm{g} / \mathrm{mL})$} \\
\hline & & $\mathrm{TO}$ & FC & GEN & GEN + TO (fold) $)^{b}$ & $\mathrm{GEN}+\mathrm{FC}(\text { fold })^{\mathrm{C}}$ \\
\hline \multicolumn{7}{|l|}{ Staphylococcus ssp. } \\
\hline S. aureus ATCC 29213 & - & $>64$ & $8-16$ & 0.5 & $0.06(8)$ & $0.06-0.12(4-8)$ \\
\hline S. aureus ATCC $29213 \Delta$ hemB (SCV) & $\mathrm{H}$ & 0.06 & 2 & 8 & - & - \\
\hline S. aureus Newbould & - & $>64$ & $8-16$ & 0.5 & $0.06(8)$ & $0.12(4)$ \\
\hline S. aureus Newbould $\Delta$ hemB (SCV) & $\mathrm{H}$ & 0.06 & 2 & 8 & - & - \\
\hline S. aureus CF07-L & - & $>64$ & 8 & 0.5 & $0.06(8)$ & $0.12(4)$ \\
\hline S. aureus CF07-S (SCV) & M & 0.12 & 2 & 8 & - & - \\
\hline S. aureus SH1000 & - & $>64$ & $8-16$ & 0.5 & $0.06(8)$ & $0.06-0.12(4-8)$ \\
\hline S. aureus MRSA USA100 ATCC BAA-41 & - & $>64$ & 16 & 0.5 & $0.12(4)$ & $0.12(4)$ \\
\hline S. aureus MRSA USA300 ATCC BAA-1556 & - & $>64$ & 16 & 0.5 & $0.06(8)$ & $0.06(8)$ \\
\hline S. aureus MRSA COL & - & $>64$ & 16 & 0.25 & $0.06(4)$ & $0.06(4)$ \\
\hline S. epidermidis ATCC 12228 & - & $>64$ & 8 & 0.5 & $0.12(4)$ & $0.12(4)$ \\
\hline \multicolumn{7}{|l|}{ Bacillus ssp. } \\
\hline B. cereus ATCC 11778 & - & $>64$ & 8 & 1 & $0.25(4)$ & $0.25(4)$ \\
\hline B. cereus ATCC 11778 (SCV\#1) & M & 0.03 & 2 & 16 & - & - \\
\hline B. cereus ATCC 11778 (SCV\#2) & M & 0.03 & 2 & 16 & - & - \\
\hline B. subtilis ATCC 6333 & - & $>64$ & 8 & 0.12 & $0.03(4)$ & $0.06(2)$ \\
\hline B. subtilis ATCC 6333 (SCV\#1) & $U$ & 0.03 & 2 & 2 & - & - \\
\hline B. subtilis ATCC 6333 (SCV\#2) & $U$ & 0.03 & 2 & 4 & - & - \\
\hline B. subtilis ATCC 9372 & - & $>64$ & 8 & 0.12 & $0.015(8)$ & $0.06(2)$ \\
\hline B. subtilis ATCC 9372 (SCV\#1) & $U$ & 0.03 & 1 & 1 & - & - \\
\hline B. subtilis ATCC 9372 (SCV\#2) & $U$ & 0.03 & 1 & 1 & - & - \\
\hline \multicolumn{7}{|l|}{ Listeria } \\
\hline L. monocytogenes ATCC 13932 & - & $>64$ & 32 & 0.5 & $0.12(4)$ & $0.12(4)$ \\
\hline L. monocytogenes ATCC 13932 (SCV\#1) & U & 0.03 & 1 & $>64$ & - & - \\
\hline L. monocytogenes ATCC 13932 (SCV\#2) & $\mathrm{H}$ & 0.03 & 1 & $>64$ & - & - \\
\hline
\end{tabular}

Abbreviations: TO tomatidine, FC FC04-100, GEN gentamicin, Auxo auxotrophy, SCV small-colony variant, $H$ hemin, $M$ menadione, $T$ thymidine, $U$ unknown ${ }^{a}$ For SCVs, auxotrophism is defined as the requirement of specific compounds in order to regain a normal growth phenotype. An agar diffusion method was used to characterize the auxotrophism of SCVs using $\mathrm{H}, \mathrm{M}$ or T. If none of these compounds restored normal growth, the auxotrophy was unknown (U)

${ }^{\mathrm{b}} \mathrm{GEN}$ was used in combination with TO at a sub-MIC of $8 \mu \mathrm{g} / \mathrm{mL}$. The fold increase in GEN susceptibility is the ratio of the MIC of GEN used alone vs GEN in the presence of TO

${ }^{\mathrm{C}} \mathrm{GEN}$ was used in combination with FC at a sub-MIC of $4 \mu \mathrm{g} / \mathrm{mL}$. The fold increase in GEN susceptibility is the ratio of the MIC of GEN used alone vs GEN in the presence of FC

FC04-100 against all prototypic Bacillales were lower than that of TO $(8-32 \mu \mathrm{g} / \mathrm{mL}$ for FC04-100 vs. $>64 \mu \mathrm{g} / \mathrm{mL}$ for TO). Furthermore, a synergy of TO or FC04-100 with GEN against all the prototypic strains tested was also observed (Table 1). Indeed, the addition of either TO or FC04-100 to GEN in the susceptibility tests provoked a 2 to 8-fold decrease of GEN MIC against all species tested (Table 1).

These results show that the spectrum of activity of TO and FC04-100 is similarly directed against the Bacillales (Staphylococcus, Bacillus, Listeria).

The interesting antibacterial activity of FC04-100 against prototypic strains was therefore noted against antibiotic multi-resistant MRSA strains (MICs of $16 \mu \mathrm{g} / \mathrm{mL}$, Table 1) and such an activity was further investigated. Although the multi-resistant $S$. aureus MRSA USA100, USA300 and $\mathrm{COL}$ are resistant to cefalexin (beta-lactam antibiotic, MIC of $256 \mu \mathrm{g} / \mathrm{mL}$ ) and to the aminoglycoside kanamycin (MIC of $256 \mu \mathrm{g} / \mathrm{mL}$ and $>1024 \mu \mathrm{g} / \mathrm{mL}$, for USA100 and USA300, respectively, Table 2), the combination of both cefalexin and kanamycin decreased their respective MICs (Table 2). Indeed, the combination of cefalexin and kanamycin in a proportion of 3:2 (commercialized under the name Ubrolexin ${ }^{\text {tr }}$ ) decreased the MIC of one or both antibiotics. This is as expected for the combination of a betalactam with an aminoglycoside [33]. However, the synergy 
Table 2 Synergistic activity of FC04-100 with the combination cefalexin:kanamycin

\begin{tabular}{llllll}
\hline & MIC $(\mu \mathrm{g} / \mathrm{mL})$ & & & \\
\cline { 2 - 5 } Species and strains & CEF & KAN & CEF:KAN $^{\mathrm{a}}$ & ${\text { CEF:KAN }(+F C)^{b}}^{\text {Fold }}$ \\
\hline S. aureus MRSA USA100 ATCC BAA-41 & 256 & 256 & $128: 86$ & $4: 3$ & 32 \\
S. aureus MRSA USA300 ATCC BAA-1556 & 256 & $>1024$ & $256: 171$ & $8: 6$ & 32 \\
S. aureus MRSA COL & 256 & 4 & $4: 3$ & $0.5: 0.35$ \\
\hline
\end{tabular}

Abbreviations: CEF cefalexin, KAN kanamycin, FC FC04-100

${ }^{a}$ CEF:KAN was used at a proportion of $3: 2$

${ }^{\mathrm{b}} \mathrm{FC} 04-100$ was used at a sub-MIC of $4 \mu \mathrm{g} / \mathrm{mL}$

'The fold increase in susceptibility is the ratio of the MIC of CEF:KAN alone vs CEF:KAN in the presence of FC04-100

is weak with MRSA strains that are resistant to one or the other or both antibiotics and the addition of $4 \mu \mathrm{g} / \mathrm{mL}$ of FC04-100 further improved by 8 to 32 fold the MIC of the cefalexin:kanamycin combination against these strains (Table 2).

\section{Bactericidal activity of TO and analog FC04-100 against} SCVs and bactericidal synergy in combination with GEN against prototypic strains

As was shown in Table 1 , the addition of $8 \mu \mathrm{g} / \mathrm{mL}$ of TO to GEN reduced the MIC of GEN against all prototypic Bacillales. In this section, kill kinetics also show that the TO-GEN combination importantly improves the bactericidal effect of GEN against prototypic B. subtilis and $B$. cereus with a $>4 \log 10$ reduction in viable bacteria at $24 \mathrm{~h}$ compared to the no drug control (Fig. 3a and b, respectively). However, although the MIC of GEN was improved when in combination with $\mathrm{TO}$ against
L. monocytogenes (Table 1), time-kill experiments failed to demonstrate a bactericidal synergy with an improvement of at most $1 \log _{10}$ in killing compared to that achieved with GEN alone (Fig. 3c). Similarly, the effect of TO used alone against SCVs was strongly bactericidal against $S$. aureus, $B$. subtilis and $B$. cereus, but not against the $L$. monocytogenes SCV (Fig. 3d), and this even if the MIC of TO against $L$. monocytogenes SCVs was $0.03 \mu \mathrm{g} / \mathrm{mL}$ (Table 1 ).

On the other hand, the addition of $8 \mu \mathrm{g} / \mathrm{mL}$ of FC04100 to GEN $(2 \mu \mathrm{g} / \mathrm{mL})$ decreased by an average of $5 \log _{10}$ the viable counts $(\mathrm{CFU} / \mathrm{mL})$ of prototypic L. monocytogenes, after $24 \mathrm{~h}$ of treatment, in comparison to GEN alone (Fig. 4a). Also, the bactericidal activity of FC04-100 used alone against the L. monocytogenes SCV was shown to be better than that observed for TO. To quantitate and facilitate such a comparison between TO and FC04-100, Fig. 4b shows the residual CFU of the L. monocytogenes SCV strain after treatment with TO or FC04-100 and
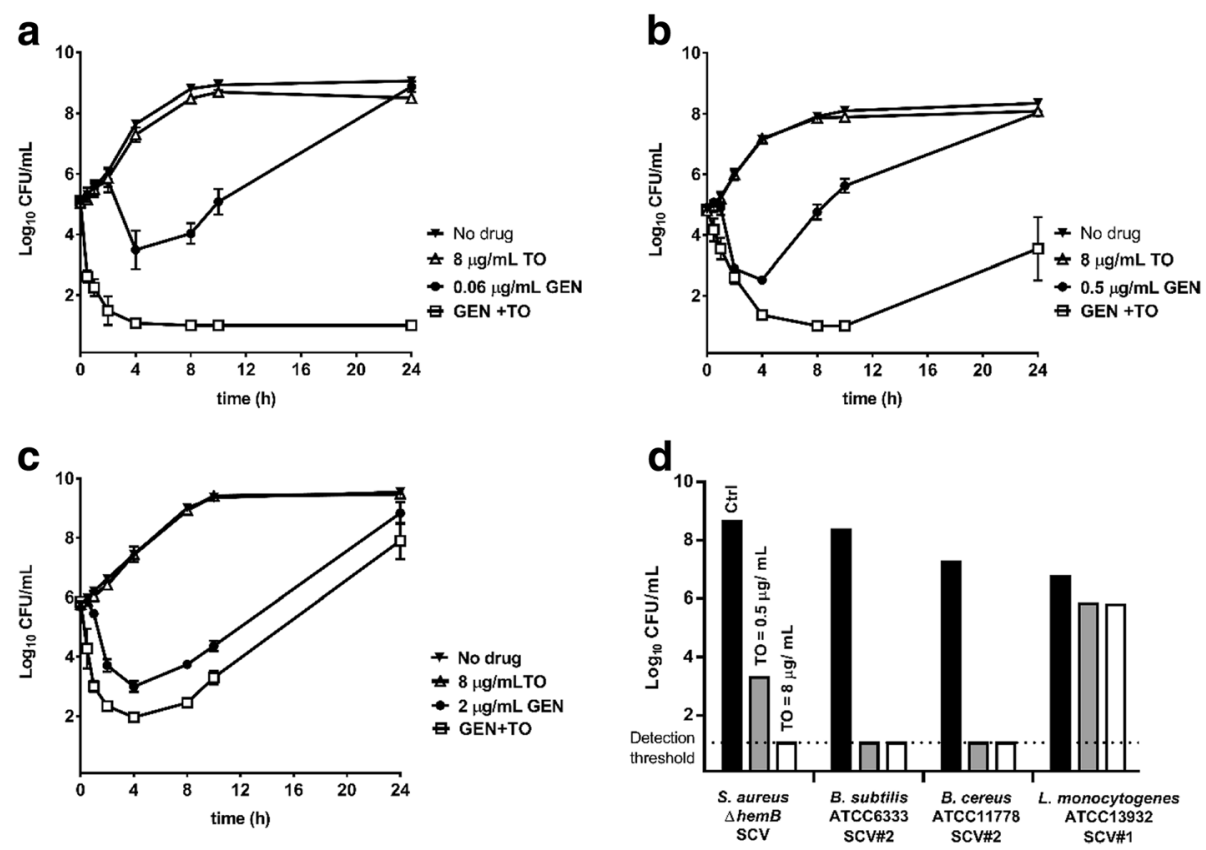

Fig. 3 Kill kinetics of TO, GEN or the combination of both (GEN + TO) against (a) prototypic B. subtilis ATCC 6333, (b) B. cereus ATCC 11778, and (c) L. monocytogenes ATCC 13932. The bactericidal effect of TO against SCVs at 0, 0.5 and $8 \mu \mathrm{g} / \mathrm{mL}$ at $24 \mathrm{~h}$ is also shown (d). The results were obtained from at least three independent experiments 


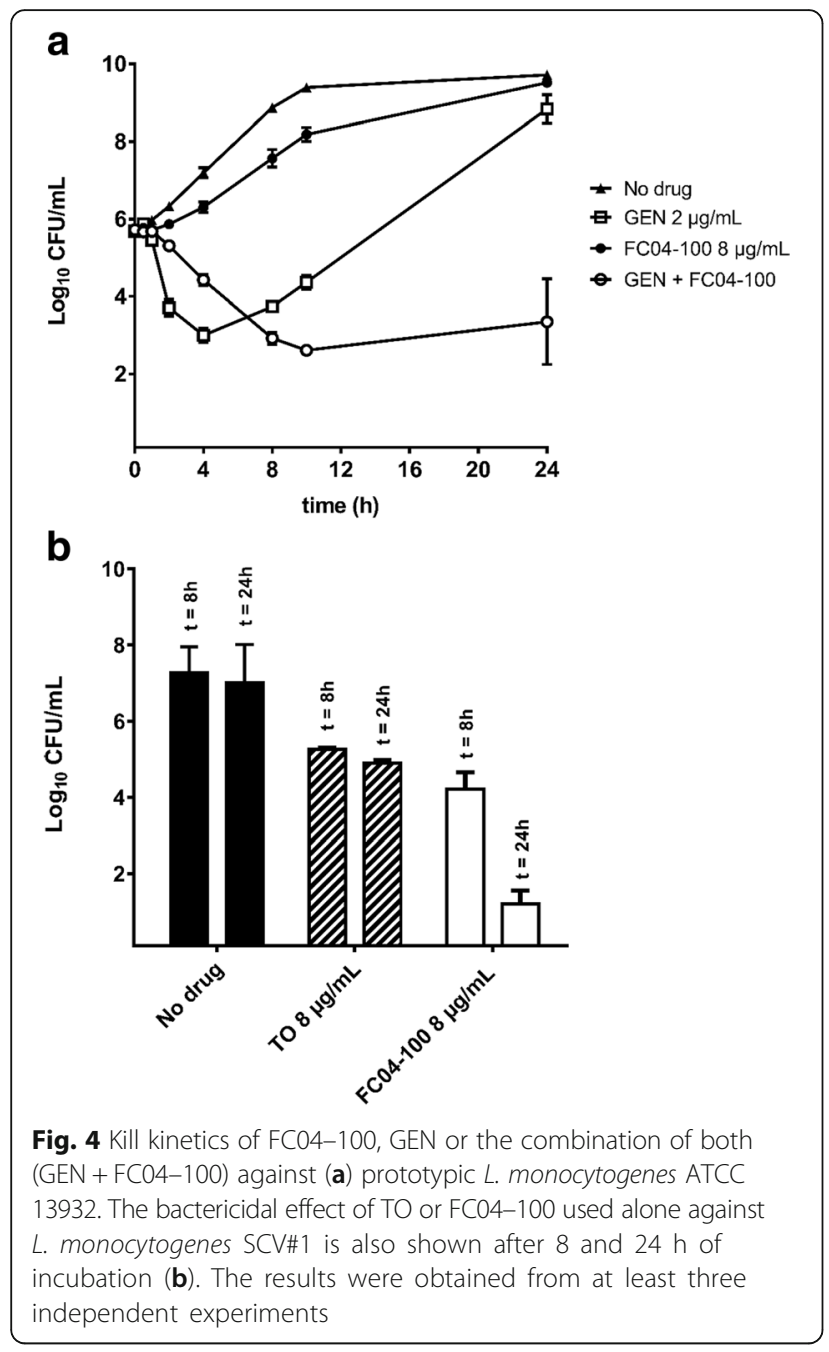

calculated as the percentage of live CFU in comparison with untreated L. monocytogenes SCVs after $24 \mathrm{~h}$ of incubation. These results show that $\mathrm{TO}$ can be chemically modified (e.g., derivative FC04-100) to improve its antibacterial activity against $L$. monocytogenes.

\section{FC04-100 potentiates the activity of GEN against}

\section{S. aureus embedded in biofilm}

The interesting bactericidal properties of FC04-100, notably against Listeria (Fig. 4) prompted us to further investigate the bactericidal activity of this steroidal alkaloid in other instances where Bacillales spp. are difficult to kill. For example, strain S. aureus SH1000 produces copious amounts of biofilm and is thus suitable for use in an assay measuring the intrabiofilm bactericidal activity of antibiotics. Figure 5 demonstrates that compound FC04-100 can also kill prototypical S. aureus SH1000 embedded in a biofilm. Indeed, FC04-100 used alone at $4 \mu \mathrm{g} / \mathrm{mL}$ (i.e., $0.25-0.5 \times \mathrm{MIC}$ ) significantly reduced the number of viable bacteria remaining in the biofilm as

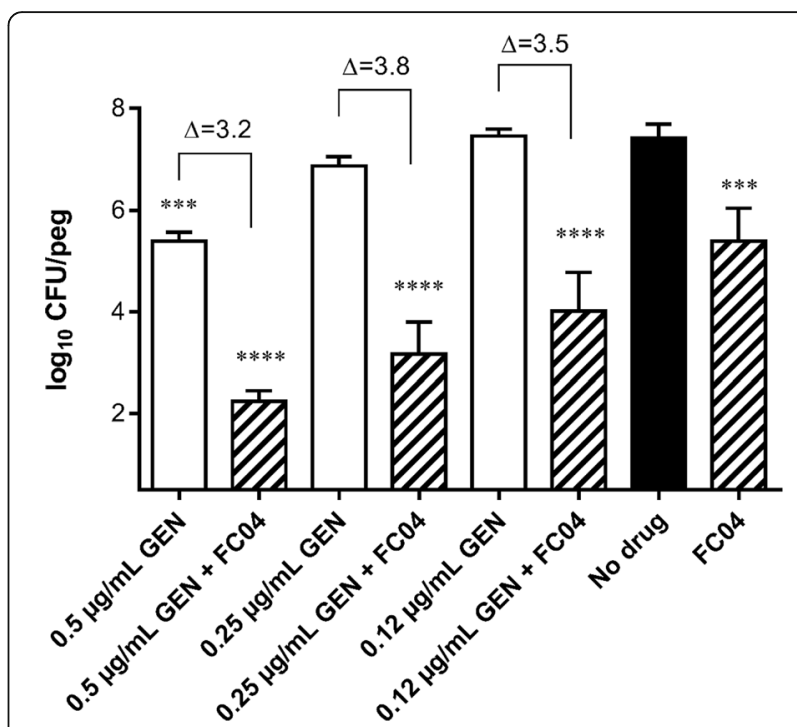

Fig. 5 Bactericidal effect of FC04-100 alone or in combination with GEN against prototypic $S$. aureus $\mathrm{SH} 1000$ embedded in a biofilm.

FC04-100 (FC04) was used at $4 \mu \mathrm{g} / \mathrm{mL}$ alone or in combination with GEN at the indicated concentrations. Data are from three independent experiments, each performed in triplicate. Significant differences in comparison to the no drug control are shown (***, $P<0.001$ and ${ }^{* * * *}$, $P<0.0001$; one-way ANOVA with a Dunnett's post test). Data are presented as means with standard deviations

compared to the no drug control $(P<0.001)$. Furthermore, when FC04-100 $(4 \mu \mathrm{g} / \mathrm{mL})$ was used in combination with GEN, the antibacterial activity of GEN (used at either $0.25,0.5$ or $1 \times \mathrm{MIC}$ ) was significantly improved $(P<0.0001)$. In other words, addition of FC04-100 to GEN improved its intrabiofilm bactericidal activity by more than $3 \log 10$ at all tested GEN concentrations (Fig. 5).

\section{FC04-100 inhibits the intracellular replication of SCVs}

Another instance where some Bacillales spp. are difficult to kill by antibiotics is when they hide within host cells. Indeed, intracellular replication of S. aureus SCVs is an important contributor to the establishment of chronic infections such as those occurring in the lungs of cystic fibrosis patients. Hence, to mimic the human pulmonary epithelium, Calu-3 cells were cultivated in an air-liquid interface and were subsequently infected with the S. aureus SCV strain CF07-S. Strain CF07-S was originally isolated from a patient with cystic fibrosis. After invasion of cells by CF07-S, remaining extracellular bacteria were removed and Calu-3 cells were exposed to TO or FC04-100 to evaluate their ability to kill intracellular bacteria. Figure 6 shows that FC04-100 (used at $8 \mu \mathrm{g} / \mathrm{mL}$ ), like TO, can significantly decrease the intracellular population of $S$. aureus CF07-S by at least $3 \log 10$ compared to the no drug control $(P<0.0001)$. 


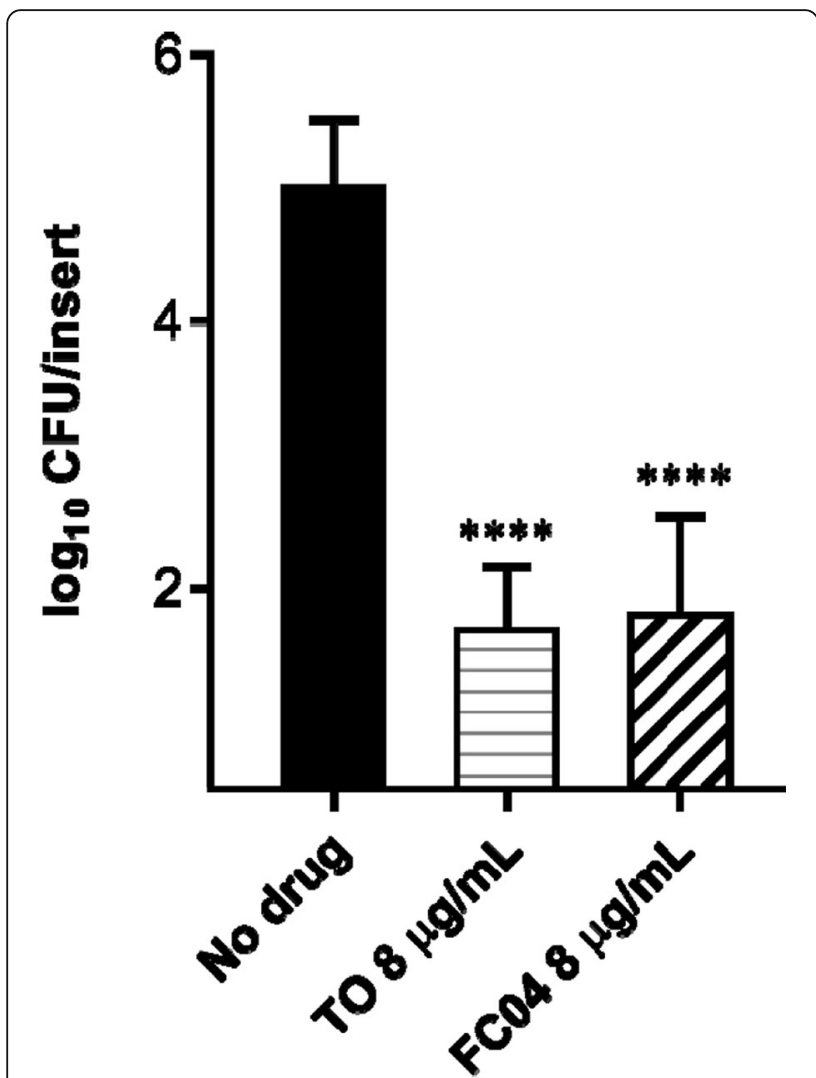

Fig. 6 Effect of TO and FC04-100 on the intracellular load of S. aureus SCV strain CF07-S $48 \mathrm{~h}$ post-internalization. Data are from three independent experiments performed in duplicate. Significant differences among groups are shown (****, $P<0.0001$, one-way ANOVA with Turkey's post test). Data are presented as means with standard deviations

\section{Discussion}

In previous studies, we have demonstrated that $\mathrm{TO}$ has a strong inhibitory activity against $S$. aureus SCVs and improves the bactericidal activity of aminoglycoside antibiotics against prototypic $S$. aureus, and also more broadly, against staphylococci. The mode of action of TO and its analog FC04-100 against S. aureus has yet to be understood. Our initial work suggested that TO inhibits the biosynthesis of macromolecules, with a pronounced effect on protein synthesis and that when used in combination, the aminoglycoside or TO could help each other to reach their respective intracellular target through cell permeabilization [19, 20]. However, more recently, we have putatively identified the cellular target of $\mathrm{TO}$ as the bacterial ATP synthase using genomic analysis of TO-resistant mutants [34]. Such a cellular target would explain both the action of TO against the already impaired respiratory-deficient SCVs, the reduction in macromolecular biosyntheses resulting from a reduction in ATP production, and the synergy with an aminoglycoside which action is also linked to the respiratory chain and proton motive force. The TOaminoglycoside synergy was documented for several prototypical strains of diverse clinical origins including aminoglycoside-resistant $S$. aureus carrying aminoglycoside modifying enzymes. This effect occurring by a mechanism that has yet to be understood was documented for several strains of diverse clinical origins including aminoglycoside-resistant $S$. aureus carrying aminoglycoside modifying enzymes. TO showed however no synergistic effect on the activity of aminoglycosides against $P$. aeruginosa, E. coli or Enterococcus spp. In the present study, we showed that the antibacterial spectrum of TO can be extended to species of the Bacillales order having very low MICs against $L$. monocytogenes, B. cereus and B. subtillis SCVs $(0.03$ to $0.12 \mu \mathrm{g} / \mathrm{mL})$. Time-kill kinetics showed that the combination of TO and GEN creates a bactericidal synergy against prototypic strains of B. subtilis and B. cereus, similarly to that previously demonstrated against $S$. aureus. However, the mixture of TO and GEN did not demonstrate much improvement over the activity of GEN alone against prototypic L. monocytogenes in time-kill experiments. Likewise, investigations with Bacillales SCVs showed that TO efficiently killed S. aureus, B. cereus and B. subtillis but not $L$. monocytogenes SCVs despite its very low MIC and TO was thus bacteriostatic against that species. L. monocytogenes SCVs are indeed quite tolerant to the bactericidal effects of many classes of antibacterial agents [35]. Therefore, amelioration of the bactericidal activity of TO against this important pathogen, as seen with its derivative FC04-100, is an important breakthrough.

TO is a steroid alkaloid found in the Solanaceae plants $[20,21]$ and is structurally characterized by 6 rings, 12 stereogenic centers, a $3 \beta$-hydroxyl group on ring $\mathrm{A}$ and spiro-fused E, F rings in the form of an aminoketal (Fig. 1). Although we previously documented some interesting antibacterial activity for $\mathrm{TO}$, the absence of an identified cellular target makes difficult the establishment of a structure-activity relationship (SAR). In initial attempts to elucidate SAR, we prepared analogs bearing modifications on ring A of TO [21]. The addition of two ammonium groups in position $\mathrm{C} 3$ on ring $\mathrm{A}$ was highly beneficial for antibiotic activity against normal non-SCV strains and the antibacterial spectrum of activity of one of such analogs, FC04-100, is detailed in the present work. On the other hand, although FC04-100 increased its antibacterial activity against prototypical strains of the Bacillales compared to TO (Table 1), it presents a reduced activity against $S$. aureus SCVs (from a MIC of $0.03-0.12 \mu \mathrm{g} / \mathrm{mL}$ for TO to a MIC of $1-2 \mu \mathrm{g} / \mathrm{mL}$ for FC04-100). The study of Chagnon et al. [21] also demonstrated that the stereochemistry of the 3 position substitution moderately affected activity against $S$. aureus SCVs and since FC04-100 is a mixture of stereoisomers 
for that position, separation and purification of FC04100 stereoisomers will need to be done in future studies to see if one or the other stereoisomer drives the antibacterial activity against prototypical or SCV strains.

Here we demonstrated that FC04-100 was able to kill both L. monocytogenes SCVs as well as the normal phenotype, alone and in the presence of GEN, respectively (Fig. 4). Furthermore, FC04-100 showed a noticeable antibacterial activity on its own (MIC of $16 \mu \mathrm{g} / \mathrm{mL}$ ) against the MRSA strains USA100, USA300 and COL (Table 1). FC04-100 was also synergistic in combination with an aminoglycoside against such MRSA strains with a 4 to 8 fold gain in the MIC of GEN. Because MRSA strains are often multi-resistant to antibiotics and often carry aminoglycoside-modifying enzymes (AMEs), we examined the possibility of using a triple antibiotic combination. Indeed, the combination of cefalexin and kanamycin in a proportion $3: 2$ is already approved to treat bovine mastitis pathogens under the brand name of Ubrolexin $^{\text {Tx }}$ [36]. This antibiotic combination offers an extended spectrum of activity compared to each individual drug and is expected to cover both $S$. aureus, Streptococcus uberis, and E. coli [36-38]. Unfortunately, the increased frequency of livestock-associated MRSA [8-10] and frequent incidence of strains and species carrying AMEs [39] may limit the spectrum of activity of the combination of a beta-lactam and an aminoglycoside. With this in mind, we showed that the use of FC04-100 in combination with cefalexin and kanamycin improves by 32 fold the activity of this mixture against MRSA strains carrying AMEs (Table 2). Overall the addition of FC04-100 to the aminoglycoside-beta-lactam combination decreased the MICs of cefalexin and kanamycin below their resistance breakpoints, and this for all the MRSA strains tested.

S. aureus, although not primarily recognized as a typical intracellular pathogen, is able to enter and survive in host cells [40]. Moreover, the ability of S. aureus to convert to the SCV phenotype showing increased biofilm production, improved adherence to host cells and tissues as well as increased intracellular persistence allows this pathogen to cause chronic and difficult to treat infections $[41,42]$. Discovering antibacterial drugs able to act in the intracellular compartment is generally recognized as a difficult challenge. Previously, we demonstrated that steroidal alkaloids such as TO had such an ability and could act on intracellular S. aureus SCVs [19], and here, we demonstrated that this was also the case for the TO analog FC04-100 (Fig. 6). Since FC04-100 showed a good bactericidal activity against $L$. monocytogenes in vitro, it will be interesting to see if it can also kill this highly specialized intracellular pathogen [43], in future studies. In addition, we also show in the present study that FC04-100 greatly improves the bactericidal activity of aminoglycosides such as GEN against bacteria embedded in biofilms. Biofilms are recognized to be a major hindrance to antibiotic action and are responsible for persistent colonization in many diseases [44] and in the food industry [45]. The important intracellular and intrabiofilm bactericidal properties of FC04-100 certainly provides further justifications for a continued interest for this class of molecules in general.

\section{Conclusion}

We showed in this study that the spectrum of activity of TO is related to the Firmicutes division, and more precisely, to the order of the Bacillales. TO possesses an antibacterial activity against SCVs and a synergistic activity with aminoglycoside antibiotics against prototypic strains. The novel TO analog FC04-100 showed very promising new characteristics that include a much improved bactericidal activity against $L$. monocytogenes and killing of $S$. aureus when embedded in biofilms as well as when bacteria are within host cells.

\begin{abstract}
Abbreviations
AME: Aminoglycoside-modifying enzyme; Auxo: Auxotrophy; BHI: Brain-heart infusion; BHIA: Brain-heart infusion agar; CAMHB: Cation-adjusted Mueller-Hinton broth; CEF: Cefalexin; CF: Cystic fibrosis; FBS: Fetal bovine serum; FC: FC04-100; GEN: Gentamicin; KAN: Kanamycin; MHA: Mueller-Hinton agar; MHB: MuellerHinton broth; MIC: Minimal inhibitory concentration; MRSA: Methicillin-resistant S. aureus; PBS: Phosphate-buffered saline; SAR: Structure-activity relationship; SCV: Small colony variant; TO: Tomatidine; TSA: Tryptic soy agar; TSB: Tryptic soy broth
\end{abstract}

\section{Acknowledgements}

Not applicable.

\section{Funding}

This study was supported by an operation grant from Cystic Fibrosis Canada to FM and EM and by a team grant from the Fonds Québécois de la Recherche sur la Nature et les Technologies (FQRNT) to FM, KB and EM. FM also acknowledges funding for this project from the Regroupement de recherche pour un lait de qualité optimale (Op+Lait, St-Hyacinthe, Québec, Canada), which is supported by the FRQNT.

\section{Availability of data and materials}

The datasets used and/or analysed during the current study are available form the corresponding authors on reasonable request. Please note that a preliminary version of the manuscript is available on the repository site of the Université de Sherbrooke.

\section{Authors' contributions \\ Conception of idea and research design: FM, EM, KB. Conduct of research and experimentation: IG, SB, CI, FC. Data analyses: IG, SB, EB, FC, EM, FM. Drafting of manuscript: IG, FM. Review and edition of final manuscript: FM, EB. All authors read and approved the final manuscript.}

\section{Ethics approval and consent to participate}

Not applicable.

Consent for publication

Not applicable.

Competing interests

The authors declare that they have no competing interests. 


\section{Publisher's Note}

Springer Nature remains neutral with regard to jurisdictional claims in published maps and institutional affiliations.

Received: 14 March 2017 Accepted: 30 January 2018

Published online: 13 February 2018

\section{References}

1. Magalhães LNM. Antimicrobial activity of rhamnolipids against Listeria monocytogenes and their synergistic interaction with nisin. Food Control. 2013;1:138-42.

2. Guillet $C$, Join-Lambert $O$, Le Monnier A, Leclercq A, Mechaï F, MamzerBruneel $M-F$, et al. Human Listeriosis caused by Listeria ivanovii. Emerg Infect Dis. 2010;16:136-8

3. Food and Drug Administration. Bad Bug Book, Foodborne pathogenic microorganisms and natural toxins. Gram-positive bacteria. Second edition. Lampel K, Al-Khaldi S, Cahill S, editors. Silver Spring: Center for Food Safety and Applied Nutrition of the Food and Drug Administration (FDA), U.S. Department of Health and Human Services; 2012.

4. Beierlein JM, Anderson AC. New developments in vaccines, inhibitors of anthrax toxins, and antibiotic therapeutics for bacillus anthracis. Curr Med Chem. 2011;18:5083-94.

5. Vuong C, Otto M. Staphylococcus Epidermidis infections. Microbes Infect. 2002:4:481-9.

6. Witte W, Strommenger B, Stanek C, Cuny C. Methicillin-resistant Staphylococcus Aureus ST398 in humans and animals, Central Europe. Emerg Infect Dis. 2007;13:255-8.

7. Hiramatsu K, Ito T, Tsubakishita S, Sasaki T, Takeuchi F, Morimoto Y, et al. Genomic basis for Methicillin resistance in Staphylococcus Aureus. Infect Chemother. 2013;45:117-36.

8. García-Álvarez L, Holden MTG, Lindsay H, Webb CR, Brown DFJ, Curran MD, et al. Methicillin-resistant Staphylococcus Aureus with a novel mecA homologue in human and bovine populations in the UK and Denmark: a descriptive study. Lancet Infect Dis. 2011;11:595-603.

9. Graveland $H$, Duim B, van Duijkeren $E$, Heederik D, Wagenaar JA. Livestock-associated methicillin-resistant Staphylococcus Aureus in animals and humans. Int J Med Microbiol. 2011:301:630-4.

10. Leonard FC, Markey BK. Meticillin-resistant Staphylococcus Aureus in animals: a review. Vet J. 2008;175:27-36.

11. Proctor RA, von Eiff C, Kahl BC, Becker K, McNamara P, Herrmann M, et al. Small colony variants: a pathogenic form of bacteria that facilitates persistent and recurrent infections. Nat Rev Microbiol. 2006:4:295-305.

12. Bryan LE, Kwan S. Aminoglycoside-resistant mutants of Pseudomonas Aeruginosa deficient in cytochrome d, nitrite reductase, and aerobic transport. Antimicrob Agents Chemother. 1981;19:958-64

13. Moisan H, Brouillette E, Jacob CL, Langlois-Bégin P, Michaud S, Malouin F. Transcription of virulence factors in Staphylococcus Aureus small-colony variants isolated from cystic fibrosis patients is influenced by SigB. J Bacteriol. 2006;188:64-76.

14. Atalla H, Gyles C, Jacob CL, Moisan H, Malouin F, Mallard B. Characterization of a Staphylococcus Aureus small colony variant (SCV) associated with persistent bovine mastitis. Foodborne Pathog Dis. 2008;5:785-99.

15. Tuchscherr L, Medina E, Hussain M, Völker W, Heitmann V, Niemann S, et al. Staphylococcus Aureus phenotype switching: an effective bacterial strategy to escape host immune response and establish a chronic infection. EMBO Mol Med. 2011;3:129-41.

16. Mitchell G, Grondin G, Bilodeau G, Cantin AM, Malouin F. Infection of polarized airway epithelial cells by normal and small-colony variant strains of Staphylococcus Aureus is increased in cells with abnormal cystic fibrosis transmembrane conductance regulator function and is influenced by NF-KB. Infect Immun. 2011;79:3541-51.

17. Ruiz-Rubio M, Pérez-Espinosa A, Lairini K, Roldàn-Arjona T, Dipietro A, Anaya N. Metabolism of the tomato saponin a-tomatine by phytopathogenic fungi. In: Rahman A, editor. Stud. Nat. Prod. Chem. 25. Oxford: Elsevier; 2001. p. 293-326.

18. Simons V, Morrissey JP, Latijnhouwers M, Csukai M, Cleaver A, Yarrow C, et al. Dual effects of plant steroidal alkaloids on Saccharomyces Cerevisiae. Antimicrob Agents Chemother. 2006;50:2732-40

19. Mitchell G, Gattuso M, Grondin G, Marsault E, Bouarab K, Malouin F. Tomatidine inhibits replication of Staphylococcus Aureus small-Colony variants in cystic fibrosis airway epithelial cells. Antimicrob Agents Chemother. 2011;55:1937-45.
20. Mitchell G, Lafrance M, Boulanger S, Séguin DL, Guay I, Gattuso M, et al. Tomatidine acts in synergy with aminoglycoside antibiotics against multiresistant Staphylococcus Aureus and prevents virulence gene expression. J Antimicrob Chemother. 2012;67:559-68.

21. Chagnon F, Guay I, Bonin M-A, Mitchell G, Bouarab K, Malouin F, et al. Unraveling the structure-activity relationship of tomatidine, a steroid alkaloid with unique antibiotic properties against persistent forms of Staphylococcus aureus. Eur J Med Chem. 2014;80:605-20.

22. Horsburgh MJ, Aish JL, White IJ, Shaw L, Lithgow JK, Foster SJ. sigmaB modulates virulence determinant expression and stress resistance: characterization of a functional rsbU strain derived from Staphylococcus aureus 8325-4. J Bacteriol. 2002;184:5457-67.

23. Brouillette E, Martinez A, Boyll BJ, Allen NE, Malouin F. Persistence of a Staphylococcus aureus small-colony variant under antibiotic pressure in vivo. FEMS Immunol Med Microbiol. 2004:41:35-41.

24. Côté-Gravel J, Brouillette E, Obradović N, Ster C, Talbot BG, Malouin F. Characterization of a vraG mutant in a genetically stable Staphylococcus aureus small-colony variant and preliminary assessment for use as a liveattenuated vaccine against intrammamary infections. Huber VC, editor. PLoS One. 2016;11:e0166621.

25. Gomaa OA, Momtaz OA. 16 rRNA characterization of bacillus isolate and its tolerence profile after subsequent subculturing. Arab J Biotech. 2007;10:107-16.

26. Sendi P, Proctor RA. Staphylococcus aureus as an intracellular pathogen: the role of small colony variants. Trends Microbiol. 2009;17:54-8.

27. Besier S, Smaczny C, von Mallinckrodt C, Krahl A, Ackermann H, Brade V, et al. Prevalence and clinical significance of Staphylococcus aureus small-colony variants in cystic fibrosis lung disease. J Clin Microbiol. 2007:45:168-72.

28. Clinical and Laboratory Standards Institute.

29. Mitchell G, Séguin DL, Asselin A-E, Déziel E, Cantin AM, Frost EH, et al. Staphylococcus aureus Sigma B-dependent emergence of small-colony variants and biofilm production following exposure to Pseudomonas aeruginosa 4-hydroxy-2-heptylquinoline-N-oxide. BMC Microbiol. 2010;10:33.

30. Ceri H, Olson M, Morck D, Storey D, Read R, Buret A, et al. The MBEC assay system: multiple equivalent biofilms for antibiotic and biocide susceptibility testing. Methods Enzymol. 2001:337:377-85.

31. Moskowitz SM, Foster JM, Emerson J, Burns JL. Clinically feasible biofilm susceptibility assay for isolates of Pseudomonas aeruginosa from patients with cystic fibrosis. J Clin Microbiol. 2004;42:1915-22.

32. Harrison JJ, Stremick CA, Turner RJ, Allan ND, Olson ME, Ceri H. Microtiter susceptibility testing of microbes growing on peg lids: a miniaturized biofilm model for high-throughput screening. Nat Protoc. 2010;5:1236-54.

33. Davis BD. Bactericidal synergism between beta-lactams and aminoglycosides: mechanism and possible therapeutic implications. Rev Infect Dis. 1982;4:237-45.

34. Lamontagne Boulet M, Guay I, Rodrigue S, Jacques P-É, Brzezinski R, Bouarab $\mathrm{K}$, et al. Identification of mutations associated with a reduced susceptibility of Staphylococcus aureus small-colony variants to tomatidine. European Society for Clinical Microbiology and Infectious Diseases, Geneva, Switzerland. European Congress of Clinical Microbiology and Infectious Diseases, ECCMID, Abst. P0729, Copenhagen; 2015.

35. Curtis TD, Gram L, Knudsen GM. The small Colony variant of Listeria monocytogenes is more tolerant to antibiotics and has altered survival in RAW 264.7 Murine macrophages. Front Microbiol. 2016:7:1056.

36. Ganière JP, Denuault L. Synergistic interactions between cefalexin and kanamycin in Mueller-Hinton broth medium and in milk. J Appl Microbiol. 2009;107:117-25.

37. Maneke E, Pridmore A, Goby L, Lang I. Kill rate of mastitis pathogens by a combination of cefalexin and kanamycin. J Appl Microbiol. 2011;110:184-90.

38. Silley P, Goby L, Pillar CM. Susceptibility of coagulase-negative staphylococci to a kanamycin and cefalexin combination. J Dairy Sci. 2012:95:3448-53.

39. Ramirez MS, Tolmasky ME. Aminoglycoside modifying enzymes. Drug Resist Updat. 2010;13:151-71

40. Lowy FD. Is Staphylococcus aureus an intracellular pathogen? Trends Microbiol. 2000:8:341-3.

41. Garcia LG, Lemaire S, Kahl BC, Becker K, Proctor RA, Denis O, et al. Antibiotic activity against small-colony variants of Staphylococcus aureus: review of in vitro, animal and clinical data. J Antimicrob Chemother. 2013;68:1455-64.

42. Mitchell G, Malouin F. Outcome and prevention of Pseudomonas aeruginosa-Staphylococcus aureus interactions during pulmonary infections in cystic fibrosis. In: Sriramulu D, editor. Cyst. Fibros. - renewed hopes through res: InTech; 2012. 
43. Hamon M, Bierne H, Cossart P. Listeria monocytogenes: a multifaceted model. Nat Rev Microbiol. 2006;4:423-34.

44. Costerton JW, Stewart PS, Greenberg EP. Bacterial biofilms: a common cause of persistent infections. Science. 1999;284:1318-22.

45. Srey S, Jahid I, Ha S-D. Biofilm formation in food industries: a food safety concern. Food Control. 2013;31:572-85.

Submit your next manuscript to BioMed Central and we will help you at every step:

- We accept pre-submission inquiries

- Our selector tool helps you to find the most relevant journal

- We provide round the clock customer support

- Convenient online submission

- Thorough peer review

- Inclusion in PubMed and all major indexing services

- Maximum visibility for your research

Submit your manuscript at www.biomedcentral.com/submit 